\title{
Lack of Huntingtin-Associated Protein-1 Causes Neuronal Death Resembling Hypothalamic Degeneration in Huntington's Disease
}

\author{
Shi-Hua Li, ${ }^{1}$ Zhao-Xue Yu, ${ }^{1}$ Cui-Lin Li, ${ }^{2}$ Huu-Phuc Nguyen, ${ }^{1}$ Yong-Xing Zhou, ${ }^{2}$ Chuxia Deng, ${ }^{2}$ and Xiao-Jiang Li ${ }^{1}$ \\ ${ }^{1}$ Department of Human Genetics, Emory University School of Medicine, Atlanta, Georgia 30322, and ${ }^{2}$ Genetics of Development and Disease Branch, \\ National Institute of Diabetes and Digestive and Kidney Diseases, National Institutes of Health, Bethesda, Maryland 20892
}

\begin{abstract}
Huntington's disease (HD) is caused by a polyglutamine expansion in the disease protein huntingtin. The polyglutamine expansion causes huntingtin to interact abnormally with a number of proteins. However, it is unclear whether, and how, huntingtin-associated proteins are involved in the neurodegeneration in HD. Here, we show that huntingtin-associated protein-1 (HAP1), which is involved in intracellular trafficking of epidermal growth factor receptor (EGFR), is highly expressed in the hypothalamus. Mice lacking HAP1 die after birth because of depressed feeding activity. Terminal deoxynucleotidyl transferase-mediated biotinylated UTP nick end labeling staining and electron microscopic examination revealed the degeneration in hypothalamic regions that control feeding behavior. Hypothalamic degeneration was also observed in HD transgenic mice that have a significant loss of body weight. Inhibition of HAP1 expression decreases EGFR signaling and cell viability, whereas overexpression of HAP1 enhances this signaling activity and inhibits mutant huntingtin-mediated cytotoxicity. These results suggest that the effect of mutant huntingtin on HAP1 and EGFR signaling may contribute to the hypothalamic neurodegeneration and loss of body weight in HD.
\end{abstract}

Key words: hypothalamus; degeneration; EGFR; Huntington; polyglutamine; huntingtin

\section{Introduction}

Huntington's disease (HD) is characterized by progressive neurodegeneration that primarily occurs in the striatum and, as the disease progresses, extends to other brain regions including the hypothalamus (Vonsattel et al., 1985; Kremer et al., 1990, 1991). The marked degeneration (up to 90\%) of the lateral hypothalamic neurons is found in HD patients in late stages of the disease (Kremer et al., 1990, 1991). These patients often show a loss of body weight (Sanberg et al., 1981; Pratley et al., 2000). HD is caused by a polyglutamine (polyQ) expansion in N-terminal huntingtin (Htt). Expression of $\mathrm{N}$-terminal mutant $\mathrm{Htt}$ in mice results in neurological symptoms and body weight loss, followed by early death. However, obvious neurodegeneration was not found at the light microscopic level (Davies et al., 1997; Schilling et al., 1999).

Like other polyQ proteins, mutant Htt forms inclusions or aggregates (Zoghbi and Orr, 2000). Whereas the role of polyQ aggregates remains elusive, it is widely accepted that $\mathrm{N}$-terminal mutant $\mathrm{Htt}$ has a gain of function by abnormally interacting with other proteins. A number of proteins have been found to bind $\mathrm{N}$-terminal Htt, and their binding is altered by polyQ expansion (Cattaneo et al., 2001). However, little is known about the patho-

Received March 24, 2003; revised May 29, 2003; accepted June 4, 2003.

This work was supported by National Institutes of Health Grants NS36232 and AG19206. We thank Drs. He Li and Manjula Rao for assistance in the initial study.

Correspondence should be addressed to Dr. Xiao-Jiang Li, Department of Human Genetics, Emory University School of Medicine, 615 Michael Street, Atlanta, GA 30322. E-mail: xiaoli@genetics.emory.edu.

Copyright $\odot 2003$ Society for Neuroscience $\quad$ 0270-6474/03/236956-09\$15.00/0 logical relevance of the interactions between $\mathrm{Htt}$ and its interacting proteins.

Among the identified Htt-interacting proteins, Htt-associated protein-1 (HAP1) is an interesting candidate for HD neuropathology. First, it is enriched in the brain (Li et al., 1995, 1996; Gutekunst et al., 1998; Page et al., 1998; Dragatsis et al., 2000), and its binding to $\mathrm{Htt}$ is enhanced by polyQ expansion ( $\mathrm{Li}$ et al., 1995, 1998a). Second, HAP1 seems to be involved in neuronal vesicular transport through its interactions with microtubulebased transporters and vesicles (Block-Galarza et al., 1997; Engelender et al., 1997; Li et al., 1998b). Recently, HAP1 was found to be involved in the endosomal trafficking of epidermal growth factor receptor (EGFR) (Li, 2002b). EGFR is important for neuronal survival (Kornblum et al., 1998; Sibilia et al., 1998). Consistent with these important roles, mice lacking HAP1 die after birth (Chan et al., 2002), although the pathological basis of their death is not clear.

Here, we report that mice lacking HAP1 develop neuronal degeneration in the hypothalamus. We also observed degeneration in the hypothalamus in HD transgenic mice. Mutant Htt binds HAP1 and decreases its expression, leading to decreased EGFR signaling and increased cytotoxicity. These studies indicate an important role for HAP1 in hypothalamic function and suggest that the dysfunction of HAP1 and defective EGFR signaling contribute to neuronal degeneration in HD.

\section{Materials and Methods}

Antibodies and reagents used. Rabbit anti-HAP1 (Li et al., 1998a), rabbit anti-Htt (EM48) (Li et al., 1998b), and mouse anti-Htt (mEM48) (Li et 
A Wild type locus

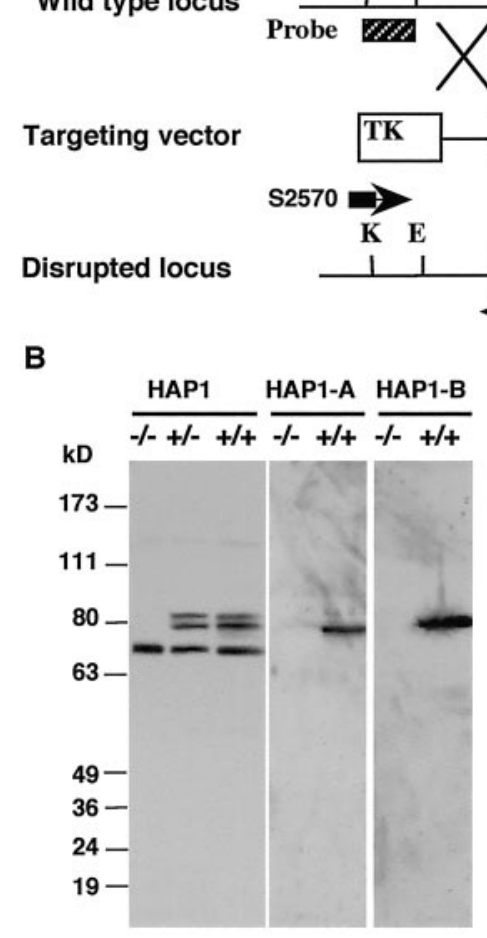

$1 \mathrm{~kb}$

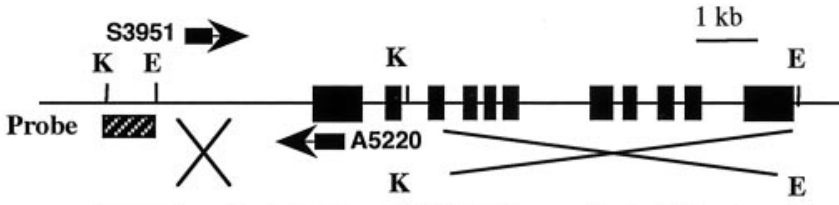

neo

HHH

$\mathbf{K}$

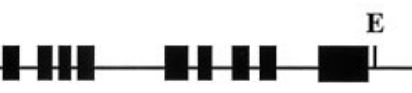

C

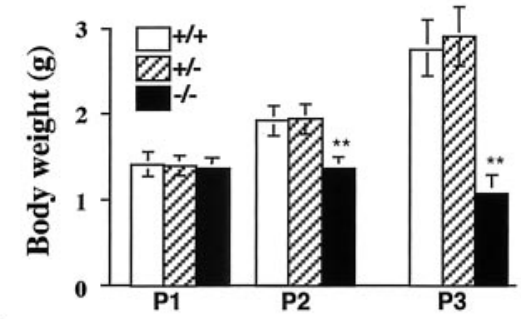

D

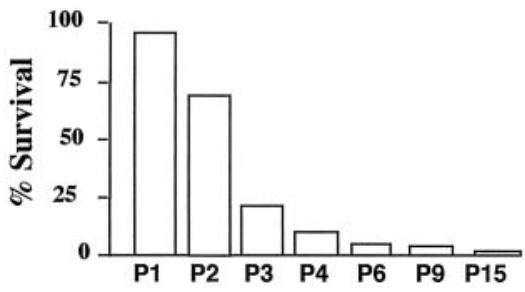

Figure 1. Targeted disruption of the mouse HAP1 gene. A, DNA structures of the wild-type allele, targeted vector, and mutant allele. The targeted vector contains neomycin (neo) and TK markers flanked by 1.6 and $5.8 \mathrm{~kb}$ of the mouse HAP1 gene. The first two exons (black boxes) of the HAP1 gene are deleted in the mutant allele. The probe used for Southern blot analysis is indicated, and the primers (arrows) for PCR genotyping are also indicated. K, Kpnl; E, ECoRI. B, Western blot analysis of brain cortex extracts of wild-type, HAP1 (+/-), and HAP1 (-/-) mice. The blots were probed with antibodies against HAP1, HAP1-A, or HAP1-B.C, Pups from HAP1 $(+/+), \operatorname{HAP} 1(+/-)$, and HAP1 $(-/-)$ mice were weighed at postnatal days 1,2 , and 3 . The data (mean \pm SEM) were obtained from $54-204$ pups for each group. ${ }^{* *} p<0.01$ compared with wild-type mice. $D$, The percentage of surviving HAP1 $(-/-)$ pups after birth. The majority of HAP1 $(-/-)$ pups died at days $3-4$ after birth.

al., 2002a) were described previously. Guinea pig anti-HAP1 (EM78) was produced by Covance (Denver, PA) using the same antigen as for rabbit anti-HAP1 (Li et al., 1995). Other antibodies used were mouse monoclonal anti-EGFR (Sigma, St. Louis, MO), anti- $\gamma$-tubulin (Sigma), antiextracellular signal-regulated kinase (ERK) (Santa Cruz Biotechnology, Santa Cruz, CA), anti-phosphorylated ERK, anti-protein kinase B (AKT), and anti-pAKT (Cell Signaling Technology, Inc., Beverly, MA). AG1478 was obtained from Calbiochem (La Jolla, CA), and other reagents from Sigma.

Homologous recombination of the HAP1 gene. Mouse genomic clones covering all 11 exons of the mouse HAP1 gene (Nasir et al., 1998) were isolated from a 129 SVJ mouse genomic DNA library (Stratagene, La Jolla, CA). A 1,575-bp fragment between EcoRI $(2,705)$ and BamHI $(4,269)$ was inserted $5^{\prime}$ of a neomycin (NeoR) and thymidine kinase (TK) cassette in the pLoxpneo vector. Another 5,792-bp fragment between KpnI $(7,126)$ and EcoRI $(13,008)$ was connected $3^{\prime}$ of the NeoR cassette. Approximately $4 \%$ of neoR/TK-selected TC-1 embryonic stem cell clones (185) were correctly targeted. Three of these were microinjected into C57BL/6 blastocysts, implanted into the uterus of pseudopregnant Swiss Webster foster mothers, and developed to term (Deng et al., 1994). Male chimeras were mated with National Institutes of Health black Swiss females (Taconic, Germantown, NY). No sex bias was observed in the offspring, and the expected Mendelian ratio (1:2:1) was observed among wild-type, heterozygous, and homozygous mutant mice. PCR genotyping of HAP1 knock-out mice used the following primers: S2570 (5'-GTGGCGCTGAGTCTGGATTGAC-3') and A4 (5'-TACCCTACCCGGTAGAATTCG-3') for the knock-out allele; S3951 (5'-TTTTGGAGGTCTGGTCTCGCTCTG-3') and A5220 (5'-CGTCTTCCATCTTAGTGCGTTCAC-3') for the wild-type HAP1 allele.

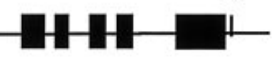

Analysis of HD mice. N171-82Q mice [B6C3F1/-TgN (HD82Gln)81Dbo], which express the first 171 amino acids of Htt with an additional 82-glutamine repeat (Schilling et al., 1999), and R6/2 mice [B6CBA-TgN (HDexon1)62], which express the first exon of $\mathrm{Htt}$ ( 67 amino acids) with an additional 115- to 150-glutamine repeat (Davies et al., 1997), were obtained from the Jackson Laboratory (Bar Harbor, ME) and bred at the Emory Animal Facility. Light and electron microscopy were performed as described previously (Li et al., 2000b). Terminal deoxynucleotidyl transferase-mediated biotinylated UTP nick end labeling (TUNEL) was performed using a kit obtained from Promega (Madison, WI) and following the manufacturer's instructions. For Western blots, the brain extracts (50-100 $\mu \mathrm{g}$ of protein) were loaded onto each lane of the SDS gel, and the immunoreactive products were visualized with ECL kits (Amersham Inc). Immunoprecipitation was performed as described ( $\mathrm{Li}$ et al., 2002a) using whole-brain extracts from R6/2 mice at 4 weeks of age and guinea pig anti-HAP1.

Analysis of transfected cells. PC12 cell lines stably expressing exon $1 \mathrm{Htt}$ containing 20- or 150-glutamine repeats were obtained from our previous study (Li et al., 1999). An anti-HAP1 construct in the PRK vector that contains the reverse sequences from the $\mathrm{N}$-terminal region of HAP1 (amino acids 1-296) was cotransfected with pCDN3 (5:1) into PC12 cells. Stably transfected PC12 cells were selected with G418 $(500 \mathrm{ng} / \mathrm{ml})$ and analyzed by Western blots to verify the decreased expression of HAP1. Of 12 cell lines isolated, we focused on the A2, A3, and A6 lines, which showed a significant decrease in HAP1 expression. Transient transfection of HEK293 cells and assays of cell viability and caspase- 3 activity were described previously (Li et al., 1999, 2002a). For EGF treatment, cells were cultured in serum-free medium overnight before EGF $(100 \mathrm{ng} / \mathrm{ml})$ was added for 5 or $45 \mathrm{~min}$.

Primary neuronal culture and transfection. Hypothalamic cells were cultured and maintained as described previously (Perez-Martinez et al., 2001) using hypothalami from embryonic days 14-16 rats. Cells were cultured in DMEM supplemented with 10\% FBS, $5 \mu \mathrm{M}$ cytosine arabinoside (Sigma), and $50 \%$ glial-conditioned medium. Neurons that had been cultured for 2- $4 \mathrm{~d}$ were transfected with HAP1-A, HAP1-B, and Htt cDNAs using CaPO4 transfection. After a 36- to 48-hr transfection, cells were analyzed. For immunofluorescent double labeling, we used mEM48 and rabbit HAP1 antibodies. We performed three to five transfection experiments for each group, examined 139-187 transfected neurons for each group, and counted neurons that showed nuclear DNA fragmentation.

Statistics. Data were analyzed using an unpaired $t$ test, with $p<0.05$ indicating statistical significance.

\section{Results}

Targeted deletion of the mouse HAP1 gene leads to postnatal death

We used a targeted gene disruption approach to eliminate the first 131 amino acids of HAP1 (Fig. $1 A$ ). Deletion of the expression of HAP1 in HAP1 $(-/-)$ mouse brain was confirmed by Western blotting with three antibodies: anti-HAP1, antiHAP1-A, and anti-HAP1-B (Fig. 1B). HAP1-A and HAP1-B, which have different C-terminal sequences ( $\mathrm{Li}$ et al., 1995) and were represented by the two top bands on the blot, were both eliminated in homozygous mutant mice (Fig. $1 B$ ).

The striking gross phenotype of $\mathrm{HAP} 1(-/-)$ mice was that 
they were unable to gain body weight after birth (Fig. 1C). Similar to HAP1 mutant mice that were generated by deleting exon 1 of the HAP1 gene (Chan et al., 2002), HAP1 (-/-) pups had very little milk in their stomachs, suggesting a feeding defect that may retard growth and cause postnatal death at days 3-4. Some female mice could produce newborn mutant mice that lived longer. However, no HAP1 $(-/-)$ mice survived $>15 \mathrm{~d}$ (Fig. 1D). In those HAP1 $(-/-)$ mice that lived longer than $9 \mathrm{~d}$, we did not observe abnormalities in movement, tactile response, motor control, skin, bones, or organs. HAP $1(+/-)$ heterozygous mice showed a similar expression level of HAP1 to wild-type mice and lived as long as wild-type mice with no obvious behavioral or body weight abnormalities. We, therefore, focused our studies on HAP1 $(-/-)$ mice to compare them with age-matched wild-type littermates.

\section{Degeneration in the hypothalamus of HAP1 $(-/-)$ mice}

We observed that in wild-type mice, HAP1 is highly expressed in the hypothalamus (Fig. 2A), including the arcuate nucleus (ARC), the paraventricular nuclei (PVN), the lateral hypothalamus (LH), and the ventromedial hypothalamic nucleus (VMN) (data not shown). The ARC receives peripheral hormone signals, such as leptin and insulin, and projects to the LH, VMN, and PVN to regulate central feeding behavior (Elmquist et al., 1998). The expression of HAP1 in the hypothalamus and the feeding phenotype of HAP1 $(-/-)$ mice are virtually the same as those of HAP1 knock-out mice reported by Chan et al. (2002). Despite the small size of the brain and body of HAP1 $(-/-)$ mice, the previous study using creyl violet or hematoxylin and eosin staining did not reveal obvious neuropathology or abnormal gross morphology in these mice (Chan et al., 2002). Our examination of the gross morphology did not reveal any significant neuropathology in the brains of $\operatorname{HAP}(-/-)$ mice either. We then used TUNEL labeling and electron microscopy to examine whether there is any abnormality in HAP1 $(-/-)$ mouse brains. TUNEL labeling clearly revealed positive cells in the hypothalamus of $\mathrm{HAP} 1(-/-)$ mice. At postnatal day 1 (P1), most TUNEL-positive cells were observed in the PVN and the LH (Fig. 2 B). Very few or no TUNELpositive cells were found in the ARC. At P3, TUNEL-labeled cells were more widely distributed in various regions of the hypothalamus, including the VMN (Fig. 2D), which also controls eating, food intake, and energy metabolism (Meguid et al., 2000). The distribution of TUNEL-positive neurons is well correlated with the high level of HAP1 in the hypothalamic nuclei.

Several other lines of evidence also suggest the presence of specific hypothalamic degeneration in $\operatorname{HAP}(-/-)$ mice. First, there were few or no TUNEL-positive cells in the hypothalamus of wild-type mouse brain at P1 (Fig. 2C). For example, quantitative analysis revealed a significant increase in TUNEL-positive cells in the HAP1(-/-) PVN (113 per image; $100 \times$ ) as compared with the PVN of wild-type pups (11 per image). Second, other brain regions such as the cortex, striatum (Fig. 2E), cerebellum, and hippocampus (data not shown) in $\operatorname{HAP} 1(-/-)$ mice had much fewer TUNEL-positive cells or showed no difference from those in wild-type control mice. Thus, deletion of HAP1 preferentially kills neurons that normally express a high level of HAP1 in the hypothalamus in mouse pups.

\section{Ultrastructural evidence for the degeneration of} hypothalamic neurons in HAP1(-/-) mice

Electron microscopy revealed that hypothalamic neurons at P1 contained degenerating mitochondria that were swollen and had
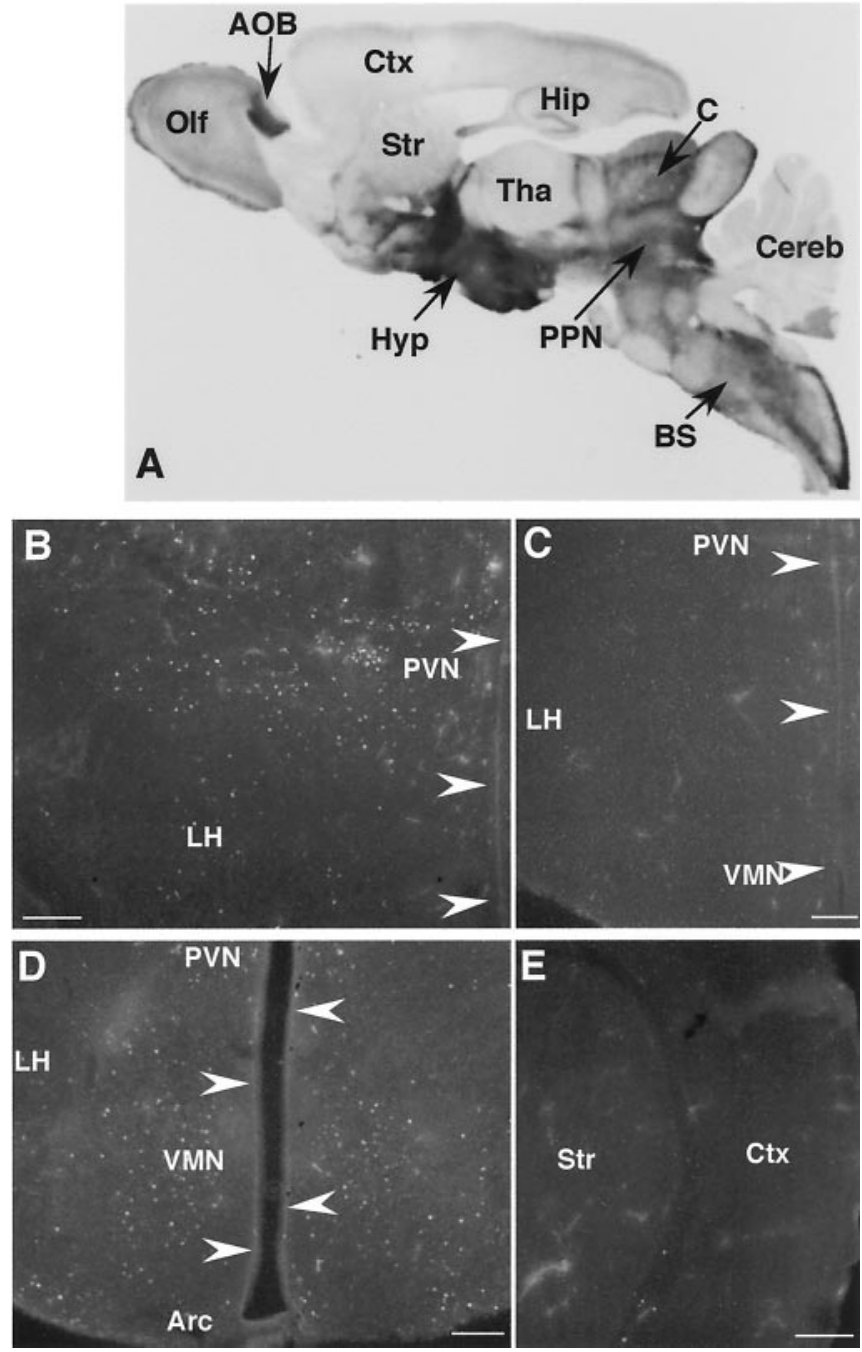

Figure 2. TUNEL staining of mouse brain. A, HAP1-B immunostaining of a sagittal section of adult mouse brain shows abundant expression of HAP1 in the hypothalamus (Hyp). HAP1 is also highly expressed in the accessory olfactory bulb (AOB), superior and inferior colliculi (C), pedunculopontine nucleus (PPN), and brain stem (BS). Olf, Olfactory bulb; Ctx, cerebral cortex; Str, striatum; Hip, hippocampus; Tha, thalamus; Cereb, cerebellum. $B-D$, Elimination of HAP1 results in TUNEL-positive cells in the hypothalamus. At P1, TUNEL-positive cells are seen in the PVN and the LH in HAP1 $(-/-)$ mice $(B)$ but not in HAP1 $(+/+)$ mice ( $C$. At P3, more TUNELpositive cells are seen in the hypothalamic areas including the VMN, whereas the ARC shows fewer TUNEL-positive cells in HAP1 $(-/-)$ mice (D). E, In the cortex of the same HAP1 $(-/-)$ mouse, no or very few TUNEL-positive cells are seen. The third ventricle is indicated by arrowheads. Scale bar, $100 \mu \mathrm{m}$.

no intact membrane structure (Fig. $3 A$ ). These abnormal morphological changes were not found in the hypothalamus of wildtype pups at P1 (Fig. 3B) or in the cortex of the same mutant pup (Fig. $3 C$ ). Some HAP1 $(-/-)$ neurons in the hypothalamus showed condensed and fragmented nuclei that constituted apoptotic bodies (Fig. 3D). This apoptotic profile was more prominent at P3-P4, as evidenced by large and multiple apoptotic bodies that were surrounded by many swollen mitochondria, large vacuoles, and lysosome-like structures (Fig. $3 E$ ). A late stage apoptotic neuron is shown in Figure $3 F$, in which an apoptotic body is engulfed by a glial cell. We did not find such degenerating neurons in the striatum or cortex of the same HAP1(-/-) mouse. Thus, electron microscopy confirms the selective degeneration of hypothalamic neurons in $\operatorname{HAP} 1(-/-)$ mice. 

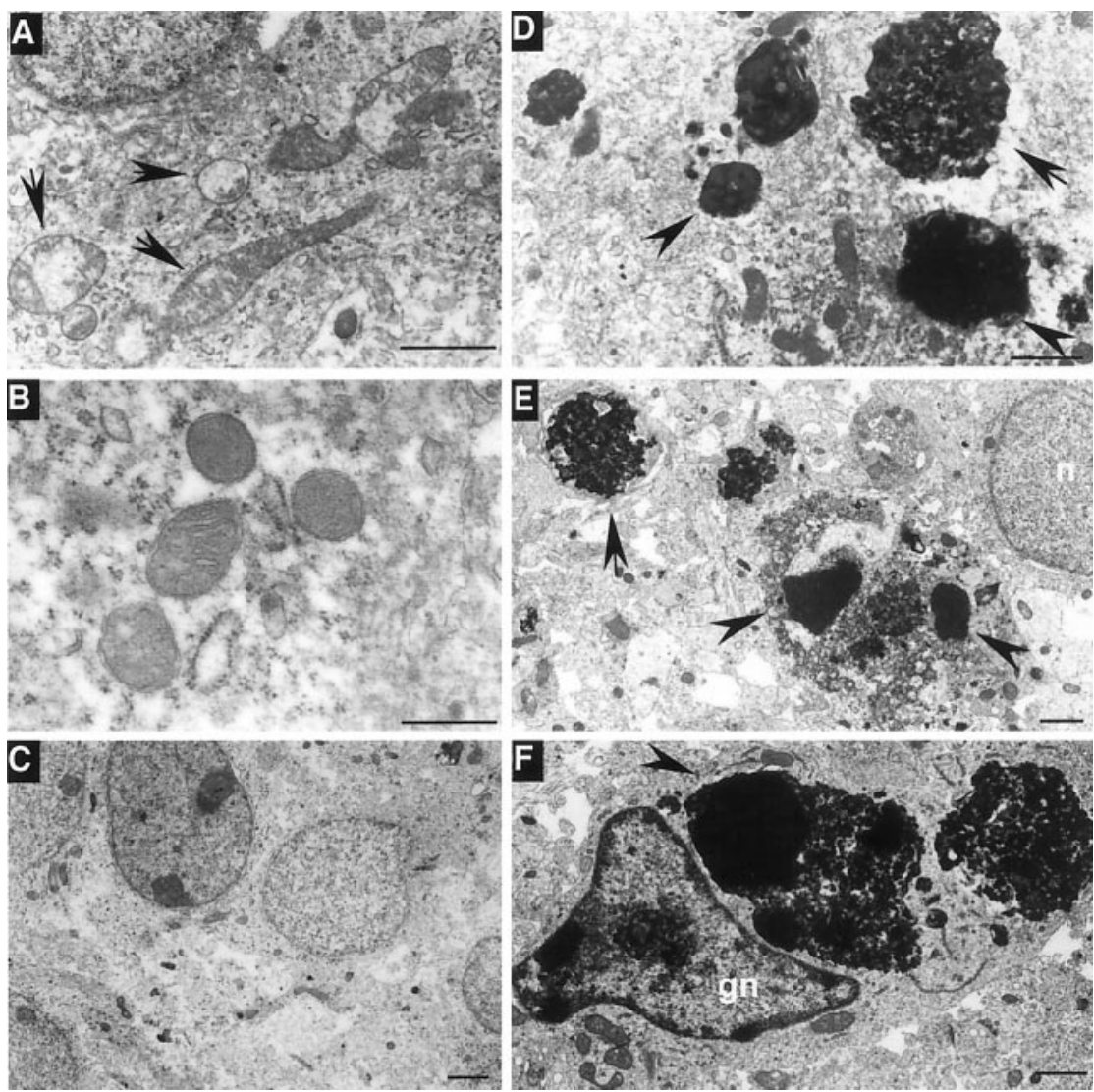

Figure 3. Electron microscopic examination of the brain of $H A P 1(-/-)$ mice. $A-D$, Ultrastructural morphology of HAP1 $(-/-)$ or $\operatorname{HAP} 1(+/+)$ mice at P1. $A$, Electron microscopy reveals swollen mitochondria with disrupted structures of internal membranes (arrows) in the LH of a HAP1 $(-/-)$ mouse. $B$, Mitochondria in the hypothalamus of a HAP1 $(+/+)$ mouse pup at $P 1$ have normal morphology. $C$, The cortex of a HAP1 $(-/-)$ mouse at $P 1$ reveals no apoptotic cells. $D$, In the hypothalamus of the same HAP1 (-/ - ) pup, fragmented and condensed nuclei form apoptotic bodies (arrowheads). $E$, $F$, Apoptotic profiles of the hypothalamic neurons of a HAP1 $(-/-)$ mouse at $\mathrm{P} 3$ showing nuclear pyknosis, chromatin fragmentation, and darkening structures (arrows). Apoptotic bodies (arrowheads) are surrounded by degenerated mitochondria and lysosome-like structures $(E)$. A normal nucleus ( $n$ ) is present near apoptotic cells $(E)$. A large apoptotic body (arrowhead) is being engulfed by a glial cell (gn), indicating a late stage of apoptosis (F). Scale bars: $A, B, 0.5 \mu \mathrm{m} ;(-F, 1 \mu \mathrm{m}$.

\section{Degeneration of hypothalamic neurons in HD transgenic mice}

Because wasting and body weight loss are typical symptoms both for HD patients (Sanberg et al., 1981; Pratley et al., 2000) and HD transgenic mice (Davies et al., 1997; Schilling et al., 1999), we examined whether there is also neuronal degeneration in the hypothalamus of N171-82Q mice that express the first 171 amino acids with a 82 -glutamine repeat $(\mathrm{Q})$. These mice have early death occurring at $5-6$ months and display a striking reduction in body weight resulting from the inability to gain weight beginning 2 months after birth (Schilling et al., 1999). Previous studies using hematoxylin/eosin and silver stains did not reveal obvious neuronal loss in these mice (Schilling et al., 1999). Using electron microscopy, we observed many degenerated neurons in the hypothalamus, as evidenced by the disrupted structures of mitochondria, condensed and dark nuclei, and some dark, electrondense cytoplasmic structures (Fig. 4A,B). To examine the distribution of mutant Htt, we also performed EM48 immunogold labeling, which showed that transgenic Htt was diffuse or scattered around the degenerated organelles (Fig. 4C). Vacuolar structures were often recruited into these degenerated organelles. In the nuclei of neurons that have not shown apoptotic bodies, mutant $\mathrm{Htt}$ formed intranuclear inclusions (Fig. 4D). Degener- ated neurons in the HD mice were present in various hypothalamic areas, including the LH, VMN, and PVN. However, other brain regions, including the cortex and striatum, also had degeneration (Yu et al., 2003), perhaps because the transgenic mutant $\mathrm{Htt}$ is widely expressed in brain. Wild-type mice at 5 months of age did not show such neurodegeneration (data not shown).

\section{Interaction of HAP1 with $\mathrm{Htt}$ and decreased expression of HAP1 and EGFR in HD mice}

The abnormal interaction between mutant Htt and HAP1 may affect the function of HAP1. To provide evidence for their interaction in vivo, we immunoprecipitated HAP1 from R6/2 HD mouse brain, which expresses mutant $\mathrm{Htt}$ at a higher level than does N171-82Q. EM48, which reacts strongly with mutant $\mathrm{Htt}$ but very weakly with endogenous rodent $\mathrm{Htt}$ ( $\mathrm{Li}$ et al., 2000b), detected the soluble and aggregated forms of mutant Htt. Both forms were precipitated by HAP1 antibody but not by preimmune serum (Fig. $5 A$ ). Probing the same blot with anti-HAP1 confirmed that Htt precipitation was specific to the presence of HAP1.

Intranuclear mutant $\mathrm{Htt}$ affects the transcriptional level of HAP1 in PC12 cells (Li et al., 1999). We performed Western blot analysis and also observed a reduced expression of HAP1 in N171-82Q mouse hypothalamus (Fig. 5B). This decrease was apparently correlated with the presence of mutant Htt. Immunohistochemistry also showed a decrease in HAP1 staining of the LH and VMN in HD mice, as compared with that in wild-type mice (Fig. 5C). This decrease was evidenced by the reduced cytosolic HAP1 staining and fewer stigmoid bodies, which are a cytoplasmic structure of unknown function and are intensively labeled by anti-HAP1 antibody (Gutekunst et al., 1998). Other brain regions that express HAP1 at a relatively lower level did not show a significant difference in HAP1 staining between wild-type and HD mice (data not shown).

Because HAP1 overexpression reduces EGFR degradation in transfected cells ( $\mathrm{Li}$ et al., 2002b), we wanted to examine the expression of EGFR in HAP1(-/-) mouse brain. Because the postnatal hypothalamus is too small to use for Western blotting, we examined the expression of EGFR in the cortex of HAP $1(-/-)$ pups. The result showed a significant decrease in the expression of EGFR (Fig. 5D). Analysis of the expression of EGFR in N171-82Q mice at 4 months of age also showed a similar decrease in EGFR expression in the hypothalamus (Fig. 5D).

\section{Decreased expression of HAP1 and overexpression of mutant Htt inhibit EGFR signaling}

The decreased expression of EGFR in HD brains prompted us to use cell models to examine whether EGFR signaling pathways are impaired by decreased HAP1 expression or increased expression 
of mutant Htt. We generated PC12 cell lines that stably express exon1 Htt containing 150Q or 20Q (Li et al., 1999). Stably transfected PC12 cells that express antisense HAP1 RNA were also established. Three cell lines (A2, A3, and A6) showed a significant decrease in the expression of HAP1 (Fig. 6A). The A2, A3, and A6 lines all had reduced neurite outgrowth in response to NGF (Fig. 6B). This result is consistent with our previous observation that overexpression of HAP1 can promote neurite outgrowth (Li et al., 2000a). We then focused on A2 cells and examined EGFR signaling, which triggers numerous downstream signaling pathways mainly through phospholipase C- $\gamma$, Ras, and phosphatidylinositol 3-kinase (PI3-kinase) to activate protein kinase $\mathrm{C}, \mathrm{ERK} 1 / 2$ cascade, and protein kinase B (AKT), respectively (Wells, 1999). Compared with nontransfected PC12 cells, A2 cells showed much less phosphorylation of ERK and AKT after EGF stimulation (Fig. 6C). This decrease was apparently associated with the reduced expression of EGFR. A similar decrease in EGFR, phosphorylated ERK, and AKT was also observed in $150 \mathrm{Q}$ cells, but not in 20Q cells, suggesting that defective EGFR signaling pathways were presented in both A2 and 150Q cells.

The impaired EGFR signaling could confer increased sensitivity of A2 and 150Q cells to EGFR signaling inhibitors. We, therefore, treated these cells with AG1478, the specific EGFR tyrosine kinase inhibitor, and wortmannin, an inhibitor of PI3-kinases. A2 and 150Q cells were more sensitive than wild-type PC12 cells to the cytotoxic effect of these inhibitors and, thus, displayed a greater decrease in their viability. The $150 \mathrm{Q}$ cells had the greatest reduction of viability in response to the highest dose of wortmannin (100 nM) (Fig. 6D), perhaps because $150 \mathrm{Q}$ activates multiple pathological pathways. Decreased viability of 20Q cells was seen with 100 nM wortmannin, suggesting that $\mathrm{N}$-terminal $\mathrm{Htt}$, when overexpressed, could also interfere with cellular function to some extent. The similar decrease in the viability of A2 and 150Q cells supports the idea that mutant Htt affects EGFR signaling activity in the same manner as the dysfunction of HAP1.

\section{Protection of HAP1 against Htt toxicity}

If mutant Htt affects the function of HAP1, overexpression of HAP1 may reduce Htt-mediated toxicity. We used transfected HEK293 cells to test this hypothesis. As reported previously (Li et al., 1999), we also observed that overexpression of HAP1 reduced the degradation of EGFR induced by EGF. This prevention, however, was inhibited by coexpression of N-terminal Htt (1-208 amino acids) containing 120Q (N208-120Q), but not by the same N-terminal huntingtin with a normal repeat (N208-23Q) (Fig. 7A). Consistently, the phosphorylation of ERK was enhanced by HAP1 overexpression and decreased by coexpression of N208-120Q. We then examined whether overexpression of HAP1 could protect against Htt toxicity in response to wortmannin, which promotes EGFR degradation and kills neurons by a caspase-dependent mechanism (Chen et al., 1999; Orike et al.,
2001). We measured caspase-3 activity, because this assay is sensitive to huntingtin toxicity in cultured cells. Wortmannin significantly increased caspase-3 activity in HEK293 cells transfected with N208-120Q. This increase, however, was inhibited by coexpression of HAP1 (Fig. $7 B$ ).

It would be interesting to see whether HAP1 also protected hypothalamic neurons from Htt toxicity. We transfected primary hypothalamic neurons with HAP1 and the same mutant Htt as described previously. Transfected HAP1-A formed small cytoplasmic inclusions resembling stigmoid bodies, but had no affect on cell viability (Fig. 7C). Expression of mutant Htt, however, resulted in the formation of large aggregates in the cytoplasm and neurites. Nuclear DNA fragmentation (Fig. 7C) and neuritic degeneration (data not shown) were often seen in mutant Htttransfected cells. When HAP1 (HAP1-A or HAP1-B) was cotransfected with mutant $\mathrm{Htt}$ into cultured neurons, both transfected HAP1 and Htt were diffusely distributed in the cell body and neurites. Some Htt aggregates in the neurites also contained HAP1 immunoreactive product, suggesting a colocalization of mutant Htt and HAP1 (Fig. 7D). Because the majority of HAP1 was diffuse in the transfected cells, most HAP1 was likely to bind to mutant $\mathrm{Htt}$ that had not formed microscopic aggregates. Importantly, neurons cotransfected with HAP1 often showed normal cellular morphology without obvious DNA fragmentation. We observed that $36.3 \%$ of mutant Htt-transfected cells showed nuclear DNA fragmentation, which is higher than that of normal Htt-transfected cells (10.1\%) or HAP1-transfected cells (6.9\%). When mutant Htt was cotransfected with HAP1, this percentage was reduced to $19.9 \%$ (Fig. $7 E$ ). These results also demonstrated 


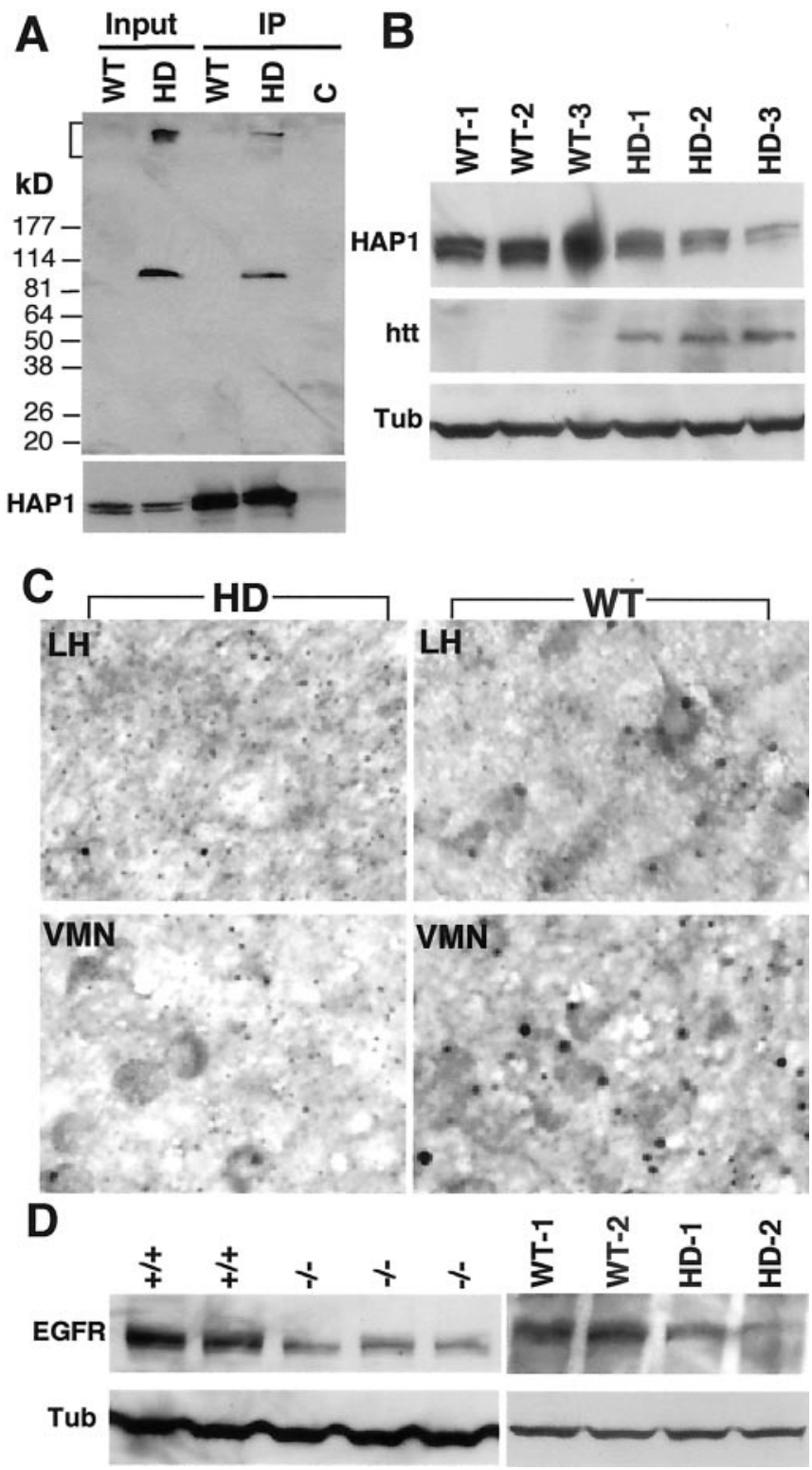

Figure 5. Expression of HAP1 and EGFR in HD transgenic mice. $A$, Immunoprecipitation of HAP1 and Htt from brains of wild-type mice (WT) and R6/2 mice (HD) at 4 weeks of age. Anti-HAP1 or the preimmune serum (lane C) was used for immunoprecipitation, and the precipitates were blotted and probed with mEM48. The bottom panel is the same blot reprobed with anti-HAP1 antibody. Input, $20 \%$ of tissue lysates. The bracket indicates the stacking gel in which aggregated $\mathrm{Htt}$ is retained. $B$, Western blot analysis of the expression of HAP1, transgenic huntingtin (htt), and tubulin shows that the expression of HAP1 is lower in the hypothalamic tissue from three different N171-820 mice (HD-1, -2, -3) than in that from wild-type mice (WT-1, -2,-3). C, HAP1 immunocytochemistry of the LH and VMN of N171-82Q and wild-type (WT) mice at 4 months of age. D, Western blot analysis of brain cortical tissues from HAP1 $1+/+)$ and HAP1 $(-/-)$ pups at P1 and the hypothalamic tissues from two wild-type mice (WT) or N171-820 mice (HD) at 4 months of age. The blots were probed with antibodies to EGFR and tubulin (Tub).

that coexpression of HAP1 can inhibit the cell death caused by mutant Htt.

\section{Discussion}

Eating behavior after birth is largely dependent on the activation of NPY signaling pathways in hypothalamic neurons by hunger signals, such as those caused by active depletion of body fat stores and/or reduced leptin/insulin signaling to the brain (Kalra et al.,
A

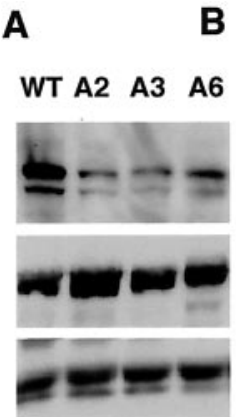

B

C
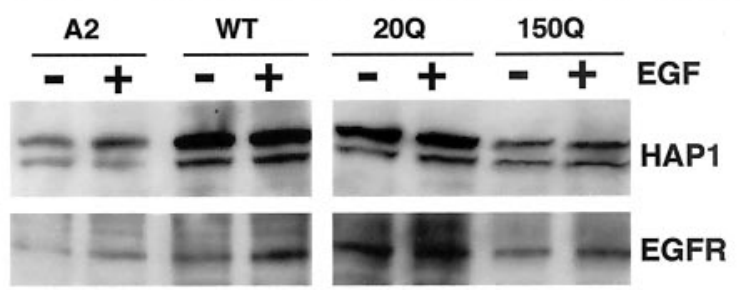

EGFR
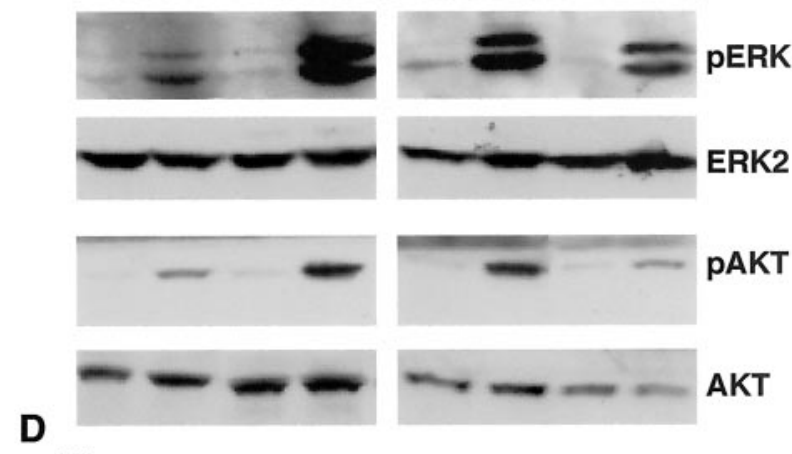

D

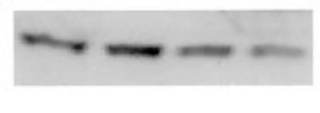

AKT
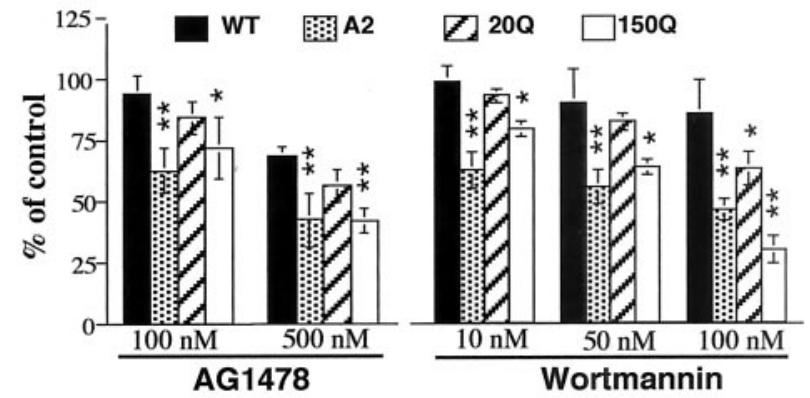

Figure 6. Decreased EGFR signaling activity in $\mathrm{PC} 12$ cells expressing antisense HAP1 or mu$\operatorname{tant} \mathrm{Htt}$. $A$, Western blot analysis of $\mathrm{PC} 12$ cell lines ( $\mathrm{A} 2, \mathrm{~A} 3$, and $\mathrm{A} 6)$ that were stably transfected with antisense HAP1 construct. WT, Wild-type PC12 cells. The expression of HAP1 (top), dynactin p150 (middle), and tubulin (bottom) is shown. B, Neurite outgrowth of PC12 cells (wild-type and $A 2$ cell line) after NGF (100 ng/ml) treatment for $48 \mathrm{hr}$. C, Expression of HAP1, EGFR, phosphorylated ERK (pERK), ERK2, phosphorylated AKT (pAKT), and AKT in wild-type cells, A2 cells, and PC12 cells stably expressing exon1 Htt with a 150-glutamine repeat (1500) or 20glutamine repeat (200) after EGF ( $100 \mathrm{ng} / \mathrm{ml})$ treatment for $5 \mathrm{~min}$. D, The viability of wild-type, $A 2,20 Q$, and $150 Q P C 12$ cells after treatment with AG1478 and wortmannin for 5 and $8 \mathrm{hr}$. The control is untreated cells for each group. Data are expressed as mean $\pm S D(n=4) .{ }^{*} p<0.05$; ${ }^{* *} p<0.01$ compared with WT.

1999; Schwartz et al., 2000). The ARC is a major site for transducing afferent input from circulating leptin and insulin into a neuronal response (Elmquist et al., 1998; Schwartz et al., 2000). Other hypothalamic areas, including the PVN and the $\mathrm{LH}$, are richly supplied by axons from NPY/agouti-related peptide and pro-opiomelanocortin and cocaine and amphetamine-regulated transcript neurons in the ARC (Elmquist et al., 1998; Kalra et al., 1999). The abundant expression of HAP1 in various hypothalamic regions is consistent with its pivotal role for the function of 
hypothalamic neurons. The lack of HAP1 causes hypothalamic neurons to die and may lead these neurons unable to respond to hunger signals.

EGFR, when translocated in the endosome, is also functional and activates the major signaling pathways, leading to cell proliferation and survival (Wang et al., 2002). Deletion of EGFR results in postnatal neurodegeneration and death in mice (Kornblum et al., 1998; Sibilia et al., 1998). HAP1 interacts with hepatocyte growth factor-regulated tyrosine kinase substrate (Hrs), which is involved in endosome-tolysosome trafficking of EGFR, and overexpressed HAP1 protects against EGFR degradation (Li et al., 2002b). The decreased expression of HAP1 could cause a rapid degradation of EGFR in certain types of neurons and, thus, decrease its signaling activity for neuronal survival, which may contribute to the neurodegeneration in HAP1 $(-/-)$ mice. Also, HAP1 seems to possess multiple functions, such as binding to dynactin P150, which is involved in microtubule-based transport (Engelender et al., 1997; Li et al., 1998b) and neurite outgrowth (Block-Galarza et al., 1997; Li et al., 2000a). These functions could also be impaired in the absence of HAP1. Because HAP1 is highly expressed in the hypothalamic regions that control feeding behavior, deletion of HAP1 may preferentially affect neuronal function and cause degeneration in these hypothalamic regions, leading to a defect in feeding behavior. As a result, $\mathrm{HAP} 1(-/-)$ mice die earlier than $\operatorname{EGFR}(-/-)$ mice, preventing us from investigating whether HAP1 deletion also causes neurodegeneration in other brain regions in older neonatal or adult mice. The role of HAP1 in other brain regions can be better investigated in future studies with a conditional HAP1 knockout mouse model.

The function of HAP1 for hypothalamic neuronal survival and EGFR signaling may be affected by mutant Htt. Indeed, similar hypothalamic degeneration was found in HD transgenic mice that show wasting and body weight loss. Weight loss in HD patients (Sanberg et al., 1981; Pratley et al., 2000) is consistent with altered release of the growth hormones from the hypothalamus (Hayden et al., 1977) and severe neuronal degeneration in the hypothalamus of HD patients (Kremer et al., 1990, 1991). Several other lines of evidence also suggest that dysfunction of HAP1 and defective EGFR signaling occur in $\mathrm{HD}$ brains. First, immunoprecipitation showed that mutant $\mathrm{Htt}$ abnormally interacted with HAP1 in HD brain. Second, consistent with our previous findings that HAP1 expression was reduced in HD patient brains and HD cells (Li et al., 1998a, 1999), the expression of HAP1 was decreased in HD mouse brains. The decreased expression of HAP1 could be because of its abnormal interaction with mutant $\mathrm{Htt}$ in the cytoplasm and the inhibitory effect of mutant $\mathrm{Htt}$ in the nucleus on gene transcription. We found that the promoter of the mouse HAP1 gene contains Sp1binding sites (data not shown), which could be affected by nuclear Htt that binds Sp1 (Dunah et al., 2002; Li et al., 2002a). Third, the increased degradation or reduced expression of EGFR was found in the brains of HAP1 knock-out and HD mice. Consistent with the in vivo evidence, both mutant $\mathrm{Htt}$ and decreased HAP1 expression caused a similar defect in EGFR signaling pathways, making cultured cells more susceptible to EGFR signaling inhibitors. Similarly, previous studies have shown that in PC12 cells mutant Htt interferes with cellular signaling mediated by EGFR (Song et al., 2002) and EGF protects against Htt toxicity (Li et al., 1999).

Although the decreased EGFR signaling activity is associated with the decreased expression of HAP1, the loss of HAP1 may also cause other cell type-specific dysfunction that contributes to neurodegeneration in the hypothalamic regions. This may explain why in $\operatorname{HAP} 1(-/-)$ cortical tissues we did not observe 
obvious neurodegeneration, even the expression of EGFR was also decreased. Similarly, mutant Htt may also cause cells to die via different pathological pathways, such as promoting NMDA toxicity (Zeron et al., 2002), activating caspases (Gervais et al., 2002; Sanchez et al., 2003), and affecting neurotrophic factor production (Zuccato et al., 2001). Different types of neurodegeneration in HD may depend on cellular context and the abnormal interactions of mutant Htt with various proteins including Hip1, which is involved in clathrin-mediated endocytosis (Metzler et al., 2001; Rao et al., 2001), therefore, leading to a variety of neurological symptoms. For example, degeneration or dysfunction of neurons in the striatum and cortex observed in different transgenic HD mouse models (Reddy et al., 1998; Hodgson et al., 1999) could be associated with motor and behavioral abnormalities, whereas degeneration in the hypothalamus may lead to wasting and body weight loss. Consistent with this idea, environmental enrichment of R6/2 mice slows the progression of neurological symptoms but not the loss of body weight (Hockly et al., 2002). Our recent studies show that huntingtin context specifies the types of neurodegeneration (Yu et al., 2003), suggesting that the interactions of various $\mathrm{N}$-terminal huntingtin fragments with other proteins are involved in the different types of neurodegeneration in HD.

An interesting question is why hypothalamic degeneration occurs in the late stages of HD. N-terminal mutant Htt may accumulate in aged neurons when their capability to remove toxic protein products is reduced. The increased concentration of $\mathrm{N}$-terminal mutant Htt fragments is then able to cause abnormal protein interactions and altered gene expression to affect the function of Htt-interacting proteins. In support of this idea, overexpressed mutant $\mathrm{N}$-terminal Htt rapidly killed the primary cultured neurons, and its toxicity could be reduced by coexpression of HAP1. Also, it remains to be investigated whether the reduced expression or partial dysfunction of HAP1 in hypothalamic neurons in adult mice leads to the feeding and wasting phenotypes similar to those of HD. Conditional HAP1 knock-out mouse models would allow one to address this issue and to investigate whether and how HAP1 is involved in regulating food intake and energy balance in adult animals. Identification of the pivotal role of HAP1 in hypothalamic neurons and its involvement in EGFR signaling pathways may help find therapeutic targets to slow the progressive neurodegeneration in HD.

\section{References}

Block-Galarza J, Chase KO, Sapp E, Vaughn KT, Vallee RB, DiFiglia M, Aronin N (1997) Fast transport and retrograde movement of huntingtin and HAP 1 in axons. NeuroReport 8:2247-2251.

Cattaneo E, Rigamonti D, Goffredo D, Zuccato C, Squitieri F, Sipione S (2001) Loss of normal huntingtin function: new developments in Huntington's disease research. Trends Neurosci 24:182-188.

Chan EY, Nasir J, Gutekunst CA, Coleman S, Maclean A, Maas A, Metzler M, Gertsenstein M, Ross CA, Nagy A, Hayden MR (2002) Targeted disruption of huntingtin-associated protein-1 (Hap1) results in postnatal death due to depressed feeding behavior. Hum Mol Genet 11:945-959.

Chen RH, Chang MC, Su YH, Tsai YT, Kuo ML (1999) Interleukin-6 inhibits transforming growth factor-beta-induced apoptosis through the phosphatidylinositol 3-kinase/Akt and signal transducers and activators of transcription 3 pathways. J Biol Chem 274:23013-23019.

Davies SW, Turmaine M, Cozens BA, DiFiglia M, Sharp AH, Ross CA, Scherzinger E, Wanker EE, Mangiarini L, Bates GP (1997) Formation of neuronal intranuclear inclusions underlies the neurological dysfunction in mice transgenic for the HD mutation. Cell 90:537-548.

Deng CX, Wynshaw-Boris A, Shen MM, Daugherty C, Ornitz DM, Leder P (1994) Murine FGFR-1 is required for early postimplantation growth and axial organization. Genes Dev 8:3045-3057.

Dragatsis I, Dietrich P, Zeitlin S (2000) Expression of the huntingtin- associated protein 1 gene in the developing and adult mouse. Neurosci Lett 282:37-40.

Dunah AW, Jeong H, Griffin A, Kim YM, Standaert DG, Hersch SM, Mouradian MM, Young AB, Tanese N, Krainc D (2002) Spl and TAFII130 transcriptional activity disrupted in early Huntington's disease. Science 296:2238-2243.

Elmquist JK, Maratos-Flier E, Saper CB, Flier JS (1998) Unraveling the central nervous system pathways underlying responses to leptin. Nat Neurosci 1:445-450.

Engelender S, Sharp AH, Colomer V, Tokito MK, Lanahan A, Worley P, Holzbaur EL, Ross CA (1997) Huntingtin-associated protein 1 (HAP1) interacts with the p150Glued subunit of dynactin. Hum Mol Genet 6:2205-2212.

Gervais FG, Singaraja R, Xanthoudakis S, Gutekunst CA, Leavitt BR, Metzler M, Hackam AS, Tam J, Vaillancourt JP, Houtzager V, Rasper DM, Roy S, Hayden MR, Nicholson DW (2002) Recruitment and activation of caspase- 8 by the huntingtin-interacting protein Hip- 1 and a novel partner Hippi. Nat Cell Biol 4:95-105.

Gutekunst CA, Li SH, Yi H, Ferrante RJ, Li XJ, Hersch SM (1998) The cellular and subcellular localization of huntingtin-associated protein 1 (HAP1): comparison with huntingtin in rat and human. J Neurosci 18:7674-7686.

Hayden MR, Vinik AI, Paul M, Beighton P (1977) Impaired prolactin release in Huntington's chorea. Evidence for dopaminergic excess. Lancet 2:423-426.

Hockly E, Cordery PM, Woodman B, Mahal A, van Dellen A, Blakemore C, Lewis CM, Hannan AJ, Bates GP (2002) Environmental enrichment slows disease progression in R6/2 Huntington's disease mice. Ann Neurol 51:235-242.

Hodgson JG, Agopyan N, Gutekunst CA, Leavitt BR, LePiane F, Singaraja R, Smith DJ, Bissada N, McCutcheon K, Nasir J, Jamot L, Li XJ, Stevens ME, Rosemond E, Roder JC, Phillips AG, Rubin EM, Hersch SM, Hayden MR (1999) A YAC mouse model for Huntington's disease with full-length mutant huntingtin, cytoplasmic toxicity, and selective striatal neurodegeneration. Neuron 23:181-192.

Kalra SP, Dube MG, Pu S, Xu B, Horvath TL, Kalra PS (1999) Interacting appetite-regulating pathways in the hypothalamic regulation of body weight. Endocr Rev 20:68-100.

Kornblum HI, Hussain R, Wiesen J, Miettinen P, Zurcher SD, Chow K, Derynck R, Werb Z (1998) Abnormal astrocyte development and neuronal death in mice lacking the epidermal growth factor receptor. J Neurosci Res 53:697-717.

Kremer HP, Roos RA, Dingjan G, Marani E, Bots GT (1990) Atrophy of the hypothalamic lateral tuberal nucleus in Huntington's disease. J Neuropathol Exp Neurol 49:371-382.

Kremer HP, Roos RA, Dingjan GM, Bots GT, Bruyn GW, Hofman MA (1991) The hypothalamic lateral tuberal nucleus and the characteristics of neuronal loss in Huntington's disease. Neurosci Lett 132:101-104.

Li XJ, Li SH, Sharp AH, Nucifora Jr FC, Schilling G, Lanahan A, Worley P, Snyder SH, Ross CA (1995) A huntingtin-associated protein enriched in brain with implications for pathology. Nature 378:398-402.

Li XJ, Sharp AH, Li SH, Dawson TM, Snyder SH, Ross CA (1996) Huntingtin-associated protein (HAP1): discrete neuronal localizations in the brain resemble those of neuronal nitric oxide synthase. Proc Natl Acad Sci USA 93:4839-4844.

Li SH, Gutekunst CA, Hersch SM, Li XJ (1998a) Interaction of huntingtinassociated protein with dynactin P150Glued. J Neurosci 18:1261-1269.

Li SH, Hosseini SH, Gutekunst CA, Hersch SM, Ferrante RJ, Li XJ (1998b) A human HAP1 homologue. Cloning, expression, and interaction with huntingtin. J Biol Chem 273:19220-19227.

Li SH, Cheng AL, Li H, Li XJ (1999) Cellular defects and altered gene expression in PC12 cells stably expressing mutant huntingtin. J Neurosci 19:5159-5172.

Li SH, Li H, Torre ER, Li XJ (2000a) Expression of huntingtin-associated protein-1 in neuronal cells implicates a role in neuritic growth. Mol Cell Neurosci 16:168-183.

Li H, Li SH, Johnston H, Shelbourne PF, Li XJ (2000b) Amino-terminal fragments of mutant huntingtin show selective accumulation in striatal neurons and synaptic toxicity. Nat Genet 25:385-389.

Li SH, Cheng AL, Zhou H, Lam S, Rao M, Li H, Li XJ (2002a) Interaction of huntington disease protein with transcriptional activator sp1. Mol Cell Biol 22:1277-1287. 
Li Y, Chin LS, Levey AI, Li L (2002b) Huntingtin-associated protein 1 interacts with hepatocyte growth factor-regulated tyrosine kinase substrate and functions in endosomal trafficking. J Biol Chem 277:28212-28221.

Meguid MM, Fetissov SO, Blaha V, Yang ZJ (2000) Dopamine and serotonin VMN release is related to feeding status in obese and lean Zucker rats. NeuroReport 11:2069-2072.

Metzler M, Legendre-Guillemin V, Gan L, Chopra V, Kwok A, McPherson PS, Hayden MR (2001) HIP1 functions in clathrin-mediated endocytosis through binding to clathrin and adaptor protein 2. J Biol Chem 276:39271-39276.

Nasir J, Duan K, Nichol K, Engelender S, Ashworth R, Colomer V, Thomas S, Disteche CM, Hayden MR, Ross CA (1998) Gene structure and map location of the murine homolog of the Huntington-associated protein, Hap1. Mamm Genome 9:565-570.

Orike N, Middleton G, Borthwick E, Buchman V, Cowen T, Davies AM (2001) Role of PI 3-kinase, Akt and Bcl-2-related proteins in sustaining the survival of neurotrophic factor-independent adult sympathetic neurons. J Cell Biol 154:995-1005.

Page KJ, Potter L, Aronni S, Everitt BJ, Dunnett SB (1998) The expression of huntingtin-associated protein (HAP1) mRNA in developing, adult and ageing rat CNS: implications for Huntington's disease neuropathology. Eur J Neurosci 10:1835-1845.

Perez-Martinez L, Charli JL, Joseph-Bravo P (2001) Development of proTRH gene expression in primary cultures of fetal hypothalamic cells. Brain Res Dev Brain Res 130:73-81.

Pratley RE, Salbe AD, Ravussin E, Caviness JN (2000) Higher sedentary energy expenditure in patients with Huntington's disease. Ann Neurol 47:64-70.

Rao DS, Chang JC, Kumar PD, Mizukami I, Smithson GM, Bradley SV, Parlow AF, Ross TS (2001) Huntingtin interacting protein 1 Is a clathrin coat binding protein required for differentiation of late spermatogenic progenitors. Mol Cell Biol 21:7796-7806.

Reddy PH, Williams M, Charles V, Garrett L, Pike-Buchanan L, Whetsell Jr WO, Miller G, Tagle DA (1998) Behavioural abnormalities and selective neuronal loss in HD transgenic mice expressing mutated full-length HD cDNA. Nat Genet 20:198-202.

Sanberg PR, Fibiger HC, Mark RF (1981) Body weight and dietary factors in
Huntington's disease patients compared with matched controls. Med J Aust 1:407-409.

Sanchez I, Mahlke C, Yuan J (2003) Pivotal role of oligomerization in expanded polyglutamine neurodegenerative disorders. Nature 421:373-379.

Schilling G, Becher MW, Sharp AH, Jinnah HA, Duan K, Kotzuk JA, Slunt HH, Ratovitski T, Cooper JK, Jenkins NA, Copeland NG, Price DL, Ross CA, Borchelt DR (1999) Intranuclear inclusions and neuritic aggregates in transgenic mice expressing a mutant $\mathrm{N}$-terminal fragment of huntingtin. Hum Mol Genet 8:397-407.

Schwartz MW, Woods SC, Porte Jr D, Seeley RJ, Baskin DG (2000) Central nervous system control of food intake. Nature 404:661-671.

Sibilia M, Steinbach JP, Stingl L, Aguzzi A, Wagner EF (1998) A strainindependent postnatal neurodegeneration in mice lacking the EGF receptor. EMBO J 17:719-731

Song C, Perides G, Liu YF (2002) Expression of full-length polyglutamineexpanded huntingtin disrupts growth factor receptor signaling in rat pheochromocytoma (PC12) cells. J Biol Chem 277:6703-6707.

Vonsattel JP, Myers RH, Stevens TJ, Ferrante RJ, Bird ED, Richardson Jr EP (1985) Neuropathological classification of Huntington's disease. J Neuropathol Exp Neurol 44:559-577.

Wang Y, Pennock S, Chen X, Wang Z (2002) Endosomal signaling of epidermal growth factor receptor stimulates signal transduction pathways leading to cell survival. Mol Cell Biol 22:7279-7290.

Wells A (1999) EGF receptor. Int J Biochem Cell Biol 31:637-643.

Yu Z-X, Li S-H, Evans J, Pillarisetti A, Li H, Li X-J (2003) Mutant huntingtin causes context-dependent neurodegeneration in mice with Huntington's disease. J Neurosci 23:2193-2202

Zeron MM, Hansson O, Chen N, Wellington CL, Leavitt BR, Brundin P, Hayden MR, Raymond LA (2002) Increased sensitivity to N-methyl-Daspartate receptor-mediated excitotoxicity in a mouse model of Huntington's disease. Neuron 33:849-860.

Zoghbi HY, Orr HT (2000) Glutamine repeats and neurodegeneration. Annu Rev Neurosci 23:217-247.

Zuccato C, Ciammola A, Rigamonti D, Leavitt BR, Goffredo D, Conti L, MacDonald ME, Friedlander RM, Silani V, Hayden MR, Timmusk T, Sipione S, Cattaneo E (2001) Loss of huntingtin-mediated BDNF gene transcription in Huntington's disease. Science 293:493-498. 